\title{
Spontaneous Emission and Characteristics of Staggered InGaN Quantum-Well Light-Emitting Diodes
}

\author{
Ronald A Arif, Hongping Zhao, Yik-Khoon Ee, and Nelson Tansu
}

\begin{abstract}
A novel gain media based on staggered InGaN quantum wells $(\mathrm{QWs})$ grown by metal-organic chemical vapor deposition was demonstrated as improved active region for visible light emitters. Fermi's golden rule indicates that InGaN QW with step-function like In content in the well leads to significantly improved radiative recombination rate and optical gain due to increased electron-hole wavefunction overlap, in comparison to that of conventional InGaN QW. Spontaneous emission spectra of both conventional and staggered InGaN QW were calculated based on energy dispersion and transition matrix element obtained by 6-band $\boldsymbol{k} \cdot \boldsymbol{p}$ formalism for wurtzite semiconductor, taking into account valence-band-states mixing, strain effects, and polarization-induced electric fields. The calculated spectra for the staggered InGaN QW showed enhancement of radiative recombination rate, which is in good agreement with photoluminescence and cathodoluminescence measurements at emission wavelength regime of 425 and $500 \mathrm{~nm}$. Experimental results of light-emitting diode (LED) structures utilizing staggered InGaN QW also show significant improvement in output power. Staggered InGaN QW allows polarization engineering leading to improved luminescence intensity and LED output power as a result of enhanced radiative recombination rate.
\end{abstract}

Index Terms-Gain media, III-nitride, InGaN quantum wells (QWs), light-emitting diodes (LEDs), polarization field engineering.

\section{INTRODUCTION}

W ITH the recent advances in the field of III-nitride semiconductors, high performance light-emitting diodes (LEDs) and lasers emitting in the visible regime have become increasingly prevalent in medical, defense and solid-state lighting applications. Conventional III-nitride based visible active region for lasers and LEDs are primarily based on type-I InGaN quantum wells (QWs) [1]-[7]. To realize high performance conventional InGaN QW LEDs and lasers, two challenges associated with wurtzite III-nitride active regions have to be addressed: 1) the high defect density and phase segregation in high-In content InGaN QW and 2) polarization-induced electrostatic field inherent in nitride materials.

Manuscript received September 14, 2007; revised December 17, 2007. This work was supported in part by the National Science Foundation under Award 0701421, in part by the U.S. Department of Defense-Army Research Laboratory, and in part by P. C. Rossin Assistant Professorship Funds.

The authors are with the Center for Optical Technologies, Department of Electrical and Computer Engineering, Lehigh University, Bethlehem PA 18015 USA (e-mail: raa4@lehigh.edu; tansu@lehigh.edu).

Color versions of one or more of the figures in this paper are available online at http://ieeexplore.ieee.org.

Digital Object Identifier 10.1109/JQE.2008.918309

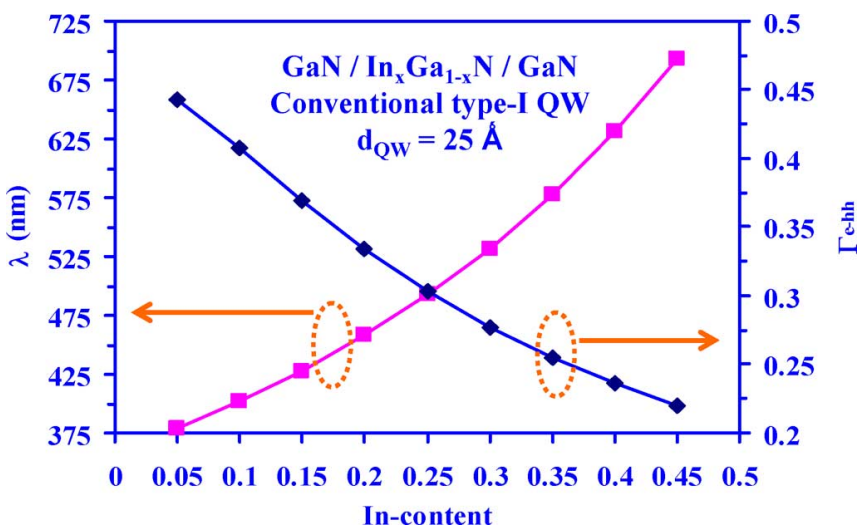

Fig. 1. Emission wavelength and electron-hole wavefunction overlap $\left(\Gamma_{\mathrm{e} \_\mathrm{h}}\right)$ for 25 - $\AA$ thick type-I $\operatorname{In}_{x} \mathrm{Ga}_{1-x} \mathrm{~N}$ QW with GaN barriers, as function of In-content.

The high threading dislocation density in III-nitrides leads to low radiative efficiency, while spontaneous and piezoelectric polarizations induce a built-in electrostatic field resulting in significant reduction of electron-hole wavefunction overlap $\left(\Gamma_{e_{-} h h}\right)[8]-[10]$. The radiative recombination rate and optical gain of the QW hence will be further reduced due to this low overlap $\Gamma_{\mathrm{e}_{-} h \mathrm{~h}}$. These challenges lead to high threshold current densities $\left(J_{\mathrm{th}} \sim 1.5-6 \mathrm{kA} / \mathrm{cm}^{2}, \lambda=425-482 \mathrm{~nm}\right)$ even in current state-of-the-art type-I InGaN QW lasers [11].

In conventional InGaN QW, the energy band bending is attributed to the inherent spontaneous and piezoelectric polarization-induced electric fields leading to quantum-confined Stark effect and spatial separation of peak electron and hole wavefunction. As a result, electron-hole wavefunction overlap $\left(\Gamma_{\text {e_hh }}\right)$ is severely reduced, in particular for thick InGaN QW with high In-content. This limitation makes realizing InGaN QW-based active region for longer wavelength emission, i.e., green and beyond, especially challenging. The impact of the internal polarization fields on the electron-hole wavefunction overlap $\left(\Gamma_{\mathrm{e} \_h h}\right)$ can be described by Fig. 1, where the emission wavelength and electron-hole wavefunction overlap $\left(\Gamma_{e_{-}}\right.$hh of 25- $\AA$ thick type-I InGaN QW as a function of In-content has been computed using our numerical model. Note that Fig. 1 was calculated without considering the carrier screening effect, thus the plot is only valid for transition wavelengths for QWs operating at carrier density from $n=5 \times 10^{18} \mathrm{~cm}^{-3}$ up to $n=1-2 \times 10^{19} \mathrm{~cm}^{-3}$ (where carrier screening is still relatively negligible). The development of our numerical model and the parameters used are described in Section II. Previously, 
the dependence of emission wavelength and transition matrix element on In-content had been shown for $30-\AA$ thick InGaN QW [12]. A similar trend is also observed in our calculation result. As the In-content in the InGaN QW is increased to extend the emission wavelength, the wavefunction overlap $\Gamma_{\mathrm{e} \_\mathrm{hh}}$ is reduced to only $27.7 \%$ for peak emission wavelength $\lambda_{\text {peak }}$ at $531.9 \mathrm{~nm}$ (yellow-green regime) due to the large internal polarization field leading to severe electron-hole separation effect. This reduction in $\Gamma_{\mathrm{e}_{-} \mathrm{hh}}$ is hence strongly detrimental in achieving low threshold current density lasers and high efficiency LEDs with InGaN QWs, in particular at high In-content.

In this work, we show that radiative recombination rate in nitride-based active region can be improved by utilizing staggered InGaN QW with step-function like In-content profile in the QW. The use of staggered InGaN QW with improved electron-hole wavefunction overlap leads to enhanced transition matrix element, which in turn leads to improved radiative recombination rate of the active regions. Both theoretical and experimental results exhibit good agreement. The implementation of the staggered InGaN QW as the active region of the LEDs also exhibits significant output power improvement [13].

It is important to note that both conventional and staggered InGaN QWs were designed for emission at a particular wavelength regime. The conventional $\mathrm{InGaN}$ QW structures were based on QW layers with thicknesses $\left(\mathrm{wc}_{1}\right)$ of $25-27 \AA$ and In-contents ( $\left.\mathrm{xc}_{1}\right)$ of $15 \%$ (for $\lambda_{\text {peak }}=420-430 \mathrm{~nm}$ ), $21 \%$ (for $\lambda_{\text {peak }}=455-465 \mathrm{~nm}$ ), and $26 \%$ (for $\lambda_{\text {peak }}=500-505 \mathrm{~nm}$ ). The staggered InGaN QW structures were subsequently optimized at each wavelength regime with improved wavefunction overlap $\left(\Gamma_{\mathrm{e}-\mathrm{hh}}\right)$, with the goal of achieving improved radiative recombination rate and radiative efficiency. The thicknesses for the staggered $\mathrm{InGaN}_{\mathrm{QWs}}\left(\mathrm{ws}_{1}, \mathrm{ws}_{2}\right.$, total thickness $=\mathrm{ws}_{1}+$ $\mathrm{Ws}_{2}$ ) with In-contents $\mathrm{Xs}_{1}$ and $\mathrm{xs}_{2}$ are not designed to be equal to those $\left(\mathrm{wc}_{1}\right)$ of the conventional InGaN QW (with In content of $\left.\mathrm{xc}_{1}\right)$, rather the choice of the parameters used in staggered structures $\left(\mathrm{xs}_{1}, \mathrm{ws}_{1}\right.$, and $\left.\mathrm{xs}_{2}, \mathrm{ws}_{2}\right)$ was determined from the numerical model calculation for optimized wavefunction overlap $\left(\Gamma_{\mathrm{e}-\mathrm{hh}}\right)$ at each particular emission wavelength.

\section{THEORETICAL BACKGROUND}

\section{A. Theory of Radiative Recombination Rate}

According to Fermi's golden rule, the electronic transition from state $|2\rangle$ to $|1\rangle$ is governed by transition matrix element via the perturbation Hamiltonian $\hat{H}_{21}^{\prime}$ [14], resulting in quantum mechanical transition rate $W_{2 \rightarrow 1}$ as follows:

$$
W_{2 \rightarrow 1}=\frac{2 \pi}{\hbar} \cdot\left|H_{21}^{\prime}\right|^{2} \rho_{f}\left(E_{1}=E_{2}-\hbar \omega_{0}\right)
$$

where $\rho_{f}$ is the density of the final states, and $\hat{H}_{21}^{\prime}$ can be expressed as a function of the transition matrix element and the envelope functions overlap. In semiconductor, the transitions occur between states in the conduction band and valence band, resulting in the following perturbation Hamiltonian

$$
\left|H_{21}^{\prime}\right|^{2} \propto \cdot\left|\left\langle u_{c}|\hat{e} \cdot \vec{p}| u_{v}\right\rangle\right|^{2} \cdot\left|\left\langle F_{2} \mid F_{1}\right\rangle\right|^{2}
$$

which depends on the square of the transition matrix element $\left|M_{T}\right|^{2}=\left|\left\langle u_{c}|\hat{e} \cdot \vec{p}| u_{v}\right\rangle\right|^{2}$ and the square of the envelope electron-hole wave function overlap $\Gamma_{\mathrm{e}_{-} \mathrm{hh}}$. The conduction and valence band Bloch functions are referred to as $\left|u_{c}\right\rangle$ and $\left|u_{v}\right\rangle$, respectively, while $\left|F_{2}\right\rangle$ and $\left|F_{1}\right\rangle$ are the envelope electron and hole wavefunction, respectively. The strength of the electric field and electron interaction is indicated by the dot product of electric field polarization $\hat{e}$ and the electron momentum $\vec{p}$. For the case of spontaneous recombination, the transition matrix element term reduces to $\left|\left\langle u_{c} \mid u_{v}\right\rangle\right|^{2}$ since the emission is not initiated by the presence of existing photons, but energy fluctuations in the vacuum state instead [15]. Both the spontaneous radiative recombination rate ( $\left.W_{\text {Spontaneous }}\right)$ and stimulated emission rate $\left(W_{\text {Stimulated }}\right)$ are then proportional to the square of the envelope electron and hole wavefunction overlap $\left|\Gamma_{\text {e_hh }}\right|^{2}$, as follows:

$$
\begin{aligned}
W_{\text {Spontaneous }} & \sim\left|\left\langle u_{c} \mid u_{v}\right\rangle\right|^{2} \cdot\left|\Gamma_{\text {e_hh }}\right|^{2} \\
W_{\text {Stimulated }} & \sim\left|\left\langle u_{c}|\hat{e} \cdot \vec{p}| u_{v}\right\rangle\right|^{2} \cdot\left|\Gamma_{\text {e_hh }}\right|^{2} .
\end{aligned}
$$

Therefore, radiative recombination rate and optical gain of III-nitride active regions can be enhanced by engineering the nanostructures with improved overlap.

\section{B. Numerical Model Development}

To design the conventional and staggered InGaN QW, we have developed a numerical model based on 6 -band $k \cdot p$ formalism for wurtzite semiconductor [16], [17]. Luminescence characteristics are studied by calculating the spontaneous recombination rate spectra, using energy dispersion relation and momentum matrix element of both the conventional and staggered InGaN QW. Effects taken into the account in the calculation include: valence band states mixing, strain, and spontaneous and piezoelectric polarization-induced electric fields. The band parameters for the III-nitride alloys were obtained from references 15-21, and these parameters are listed in Table I. The $\mathrm{GaN}$ electron effective mass constants of $0.18 m_{O}$ and $0.2 m_{O}$ were used for the $c$ axis and transverse direction, respectively [19]. The InN electron effective mass of $0.11 m_{O}$ was used for both the $c$ axis and transverse directions [19]. The heavy hole effective masses were calculated following the treatment presented in reference 18 . The ratio of conduction and valence band offsets $\Delta E_{c}: \Delta E_{v}$ is taken as 70:30 [20]. The energy gap of the InGaN QW is calculated using bowing parameter of 1.4 $\mathrm{eV}$ [18] and $\mathrm{InN}$ energy gap of $0.6405 \mathrm{eV}$ [21]. Indium concentration profile along the growth axis is incorporated into energy band lineup, with the corresponding strain taken into account as band edge energy shifts. Polarization-induced electric field is manifested in the energy band bending. The spontaneous polarization $P_{\text {sp_InGaN }}\left(C / \mathrm{m}^{2}\right)$ and piezoelectric polarization $P_{\text {pz_InGaN }}\left(C / m^{2}\right)$ in the InGaN QW are calculated using the following relations [22]:

$$
\begin{aligned}
P_{\mathrm{sp} \_ \text {InGaN }}(x)= & -0.042 \cdot x-0.034 \cdot(1-x) \\
& +0.037 \cdot x \cdot(1-x) \\
P_{\mathrm{pz} \_ \text {InGaN }}(x)= & 0.148 \cdot x-0.0424 \cdot x \cdot(1-x)
\end{aligned}
$$


with $x$ as the In-content in the $\mathrm{QW}$. The spontaneous recombination rate per unit energy per unit volume $r_{\mathrm{sp}}(\hbar \omega)$ is calculated using the fact that momentum-matrix element of the spontaneous emission is the angular average of two TE-polarization components in the transverse plane and one TM-polarization component in the $z$-direction [17]

$$
r_{\mathrm{sp}}(\hbar \omega)=\frac{n^{2} \omega^{2}}{\pi^{2} \cdot \hbar \cdot c^{2}} \cdot \frac{\left(2 \cdot g_{\mathrm{sp}}^{\mathrm{TE}}+g_{\mathrm{sp}}^{\mathrm{TM}}\right)}{3}
$$

with the $g_{\mathrm{sp}}^{\mathrm{TE}}$ or TM defined as

$$
\begin{aligned}
g_{\mathrm{sp}}^{e}(\hbar \omega)= & \frac{2 \cdot q^{2} \cdot \pi}{n \cdot c \cdot \varepsilon_{o} \cdot m_{o}^{2} \cdot \omega \cdot L_{w}} \\
& \cdot \sum_{\sigma=U, L} \sum_{n, m} \int \frac{k_{t} \cdot d k_{t}}{2 \cdot \pi} \cdot\left|\left(M_{\mathrm{e}}\right)_{n m}^{\sigma}\left(k_{t}\right)\right|^{2} \\
& \cdot \frac{f_{n}^{c}\left(k_{t}\right) \cdot\left(1-f_{\sigma m}^{v}\left(k_{t}\right)\right) \cdot\left(\frac{\gamma}{\pi}\right)}{\left(E_{n}^{c}\left(k_{t}\right)-E_{\sigma, m}^{v}\left(k_{t}\right)-\hbar \omega\right)^{2}+\gamma^{2}} .
\end{aligned}
$$

The Fermi Dirac distribution functions for electron $f_{n}^{c}$ and hole $f_{\sigma m}^{v}$ are defined as

$$
\begin{aligned}
f_{n}^{c}\left(k_{t}\right) & =\frac{1}{1+\exp \left(\frac{E_{n}^{c}\left(k_{t}\right)-F_{c}}{k_{B} \cdot T}\right)} \\
f_{\sigma m}^{v}\left(k_{t}\right) & =\frac{1}{1+\exp \left(\frac{E_{\sigma, m}^{v}\left(k_{t}\right)-F_{v}}{k_{B} \cdot T}\right)}
\end{aligned}
$$

where $E_{n}^{c}\left(k_{t}\right)$ and $E_{\sigma m}^{v}\left(k_{t}\right)$ are the eigenenergies of electron and hole, respectively. The parameters $F_{c}$ and $F_{v}$ are the carrierdensity dependent quasi-Fermi levels for electron and holes, and these terms are related to the injection carrier in the QW [14], [17]. Electron and hole concentrations in the QW are assumed to be equal, under high carrier injection. The TE- and TM-polarized matrix elements are shown below

$$
\begin{aligned}
& \left|\left(M_{\mathrm{e}}\right)_{n m}^{\sigma}\left(k_{t}\right)\right|^{2} \\
& =\frac{\left|\left\langle S\left|p_{x}\right| X\right\rangle\right|^{2}}{4} \cdot\left\{\left\langle\phi_{n} \mid g_{m}^{(1)}\right\rangle^{2}+\left\langle\phi_{n} \mid g_{m}^{(2)}\right\rangle^{2}\right\} \\
& =\frac{\left|\left\langle S\left|p_{x}\right| X\right\rangle\right|^{2}}{4} \cdot\left\{\left\langle\phi_{n} \mid g_{m}^{(4)}\right\rangle^{2}+\left\langle\phi_{n} \mid g_{m}^{(5)}\right\rangle^{2}\right\}
\end{aligned}
$$

\begin{tabular}{|c|c|c|}
\hline Parameter & GaN & InN \\
\hline $\begin{array}{c}\text { Lattice constants } \\
\mathrm{a}(\AA) \\
\end{array}$ & 3.189 & 3.548 \\
\hline \multicolumn{3}{|l|}{ Energy constants } \\
\hline $\mathrm{E}_{\mathrm{g}}(\mathrm{eV})$ & 3.42 & 0.6405 \\
\hline $\mathrm{D}_{1}=\mathrm{D}_{\mathrm{cr}}(\mathrm{eV})$ & 0.019 & 0.041 \\
\hline $\mathrm{D}_{\mathrm{so}}$ & 0.014 & 0.001 \\
\hline $\mathrm{D}_{2}=\mathrm{D}_{3}=\mathrm{D}_{\mathrm{so}} / 3(\mathrm{meV})$ & 4.67 & 0.33 \\
\hline Bowing parameter $(\mathrm{eV})$ & & 1.4 \\
\hline \multicolumn{3}{|c|}{ Conduction band effective masses } \\
\hline $\mathrm{me}_{\mathrm{z}} / \mathrm{m}_{\mathrm{o}}$ & 0.18 & 0.11 \\
\hline $\mathrm{me}_{\mathrm{t}} / \mathrm{m}_{0}$ & 0.2 & 0.11 \\
\hline \multicolumn{3}{|c|}{ Valence band effective mass parameters } \\
\hline $\mathrm{A}_{1}$ & -7.24 & -9.24 \\
\hline $\mathrm{A}_{2}$ & -0.51 & -0.6 \\
\hline $\mathrm{A}_{3}$ & 6.73 & 8.68 \\
\hline $\mathrm{A}_{4}$ & -3.36 & -4.34 \\
\hline $\mathrm{A}_{5}$ & -3.4 & -1.47 \\
\hline $\mathrm{A}_{6}$ & -4.9 & -1.64 \\
\hline \multicolumn{3}{|c|}{ Deformation potentials $(\mathrm{eV})$} \\
\hline $\mathrm{a}_{\mathrm{c}}$ & -4.08 & -4.08 \\
\hline $\mathrm{D}_{1}=\mathrm{D}_{\mathrm{cr}}$ & 0.7 & 0.7 \\
\hline $\mathrm{D}_{2}$ & 2.1 & 2.1 \\
\hline $\mathrm{D}_{3}$ & 1.4 & 1.4 \\
\hline $\mathrm{D}_{4}$ & -0.7 & -0.7 \\
\hline \multicolumn{3}{|c|}{ Elastic stiffness constants $\left(10^{11} \mathrm{dyn} / \mathrm{cm}^{2}\right)$} \\
\hline $\mathrm{C}_{13}$ & 10 & 12.4 \\
\hline $\mathrm{C}_{33}$ & 39.2 & 18.2 \\
\hline
\end{tabular}

$$
\begin{aligned}
& \left|\left(M_{z}\right)_{n m}^{\sigma}\left(k_{t}\right)\right|^{2} \\
& =\frac{\left|\left\langle S\left|p_{z}\right| Z\right\rangle\right|^{2}}{4} \cdot\left\langle\phi_{n} \mid g_{m}^{(3)}\right\rangle^{2} \quad \text { for } \sigma=U \\
& =\frac{\left|\left\langle S\left|p_{z}\right| Z\right\rangle\right|^{2}}{4} \cdot\left\langle\phi_{n} \mid g_{m}^{(6)}\right\rangle^{2} \quad \text { for } \sigma=L
\end{aligned}
$$

where $\phi_{n}$ and $g_{m}$ are conduction and valence band confined states, respectively. These confined states are the eigenvectors of the block-diagonalized $6 \times 6$ Hamiltonian matrix [16], [17]. Upper and lower Hamiltonian block are indicated by $\sigma=U$ and $\sigma=L$, respectively. Linewidth broadening with Lorentzian
TABLE I

MATERIAL PARAMETERS FOR GAN, ALN, AND INN USED IN QW BAND Structure Calculations. The Parameter VALUES ARE TAKEN FROM [16]-[22]

shape of $\gamma=0.1 \mathrm{ps}$ were used throughout the calculations. The details of the material parameters utilized in the calculation are listed in Table I. In our studies, the conventional and staggered InGaN QWs were designed for emission in a particular wavelength regime, with the staggered QWs optimized to give the largest wavefunction overlap $\left(\Gamma_{\mathrm{e} \_h h}\right.$ at the band edge $\left.k=0\right)$ in that regime.

The calculations of the spontaneous emission spectra presented in these studies do not account for carrier screening effect in the InGaN QW system, which is important in particular for carrier density above $3 \times 10^{19} \mathrm{~cm}^{-3}$. Our current studies focus on the comparison of the spontaneous emission spectra and radiative recombination rate of the conventional and staggered InGaN QW for LED operation with carrier density ranging from $5 \times 10^{18} \mathrm{~cm}^{-3}$ up to $2 \times 10^{19} \mathrm{~cm}^{-3}$, thus carrier screening is relatively negligible. The typical current density in LED devices ranges from 20 up to $200 \mathrm{~A} / \mathrm{cm}^{2}$ [23]-[25], which corresponds to the typical carrier density ranging from $2-5 \times 10^{18} \mathrm{~cm}^{-3}$ up to $1 \times 10^{19} \mathrm{~cm}^{-3}$ [25]-[27]. The current density for LED operation is approximately an order of magnitude lower than that of the threshold current density of InGaN QW lasers $\left(J_{\mathrm{th}} \sim\right.$ $1.5-2 \mathrm{kA} / \mathrm{cm}^{2}$ ) [28], [29]. The typical threshold carrier density of InGaN QW lasers approximately ranges from $4 \times 10^{19} \mathrm{~cm}^{-3}$ up to $7 \times 10^{19} \mathrm{~cm}^{-3}$ [30], where carrier screening effect is significant. Thus, carrier screening effect is important to be included in the optical gain analysis of InGaN-based QW active regions for laser devices, using self-consistent model for carrier density higher than $3 \times 10^{19} \mathrm{~cm}^{-3}$. 


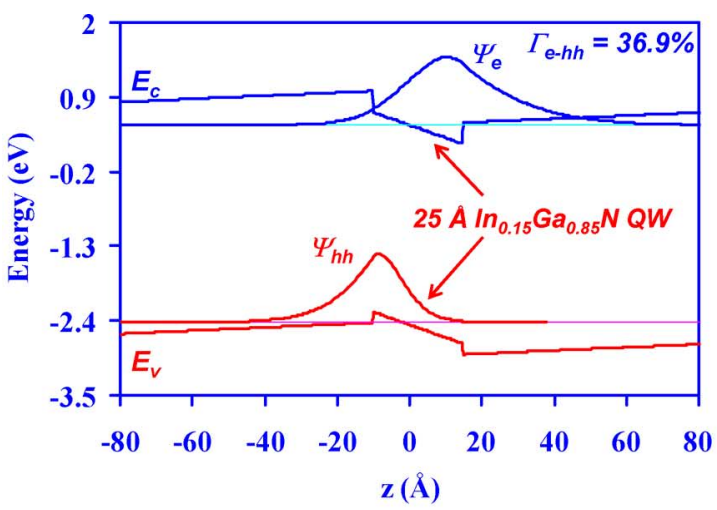

(a)

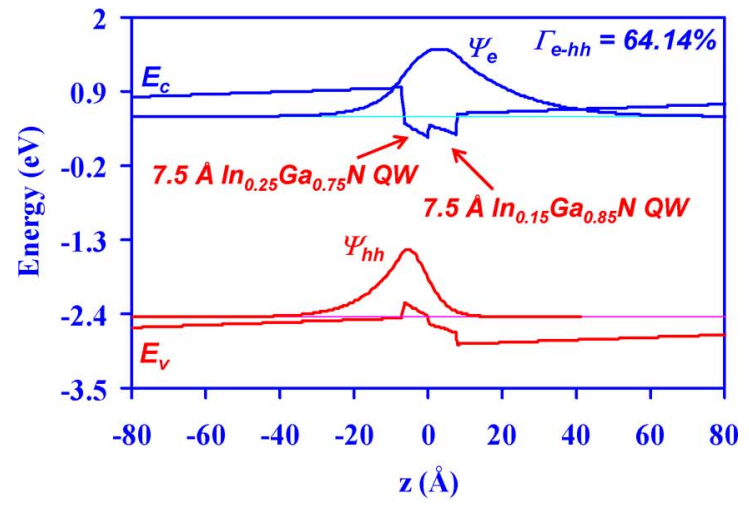

(b)

Fig. 2. Energy band lineups of (a) $25-\AA ̊$ conventional type-I $\operatorname{In}_{0.25} \mathrm{Ga}_{075} \mathrm{~N}$ QW and (b) staggered 7.5- $\AA \operatorname{In}_{0.25} \mathrm{Ga}_{0.75} \mathrm{~N}-7.5-\AA \mathrm{In}_{0.15} \mathrm{Ga}_{0.85} \mathrm{~N}$ QW.

\section{CALCUlATion AND EXPERIMENTAL RESUltS}

\section{A. Comparison in the Blue Regime $\left(\lambda_{\text {peak }}=420-430 \mathrm{~nm}\right)$}

Fig. 2 shows the band lineup of $25-\AA \mathrm{In}_{0.15} \mathrm{Ga}_{0.85} \mathrm{~N}$ QW surrounded by $\mathrm{GaN}$ barriers with its corresponding electron and hole wave functions, for emission at $\lambda_{\text {peak }}=427.5 \mathrm{~nm}$ regime. The polarization fields in the conventional $\mathrm{InGaN}$ QW lead to low wavefunction overlap $\Gamma_{e_{-} h h}$ of only $36.9 \%$. The staggered QW structures (designed for $\lambda_{\text {peak }}=421-\mathrm{nm}$ ) consist of $7.5 \AA \mathrm{In}_{0.25} \mathrm{Ga}_{0.75} \mathrm{~N}-7.5-\AA \mathrm{In}_{0.15} \mathrm{Ga}_{0.85} \mathrm{~N}$ layers surrounded by GaN barriers, as shown in Fig. 2(b). The utilization of staggered InGaN QWs leads to the "pulling" of electron wavefunction from the right to the center of the $\mathrm{QW}$, due to the lighter electron effective mass in comparison to that of the hole. The hole wave function is relatively unchanged due to the heavier hole effective mass. As a result, the wavefunction overlap $\Gamma_{\text {e-hh }}$ for the staggered InGaN QWs is increased to $64.14 \%$. An improvement of the wavefunction overlap $\Gamma_{\mathrm{e}_{-} \mathrm{hh}}$ at $k=0$ of the ground state $\left(E_{c 1}-E_{\mathrm{HH} 1}\right)$ transition by a factor of 1.74 should translate to $\sim 3.02$ times improvement in peak radiative recombination rate and optical gain of the active region at the peak wavelength.

The calculated spontaneous emission rate spectra for the corresponding conventional and staggered $\mathrm{InGaN}$ QW structures are shown in Fig. 3. The spectra were plotted for carrier densities $n$ from $5 \times 10^{18} \mathrm{~cm}^{-3}$ up to $2 \times 10^{19} \mathrm{~cm}^{-3}$. The carrier densities $n$ refers to the densities of the injected carrier

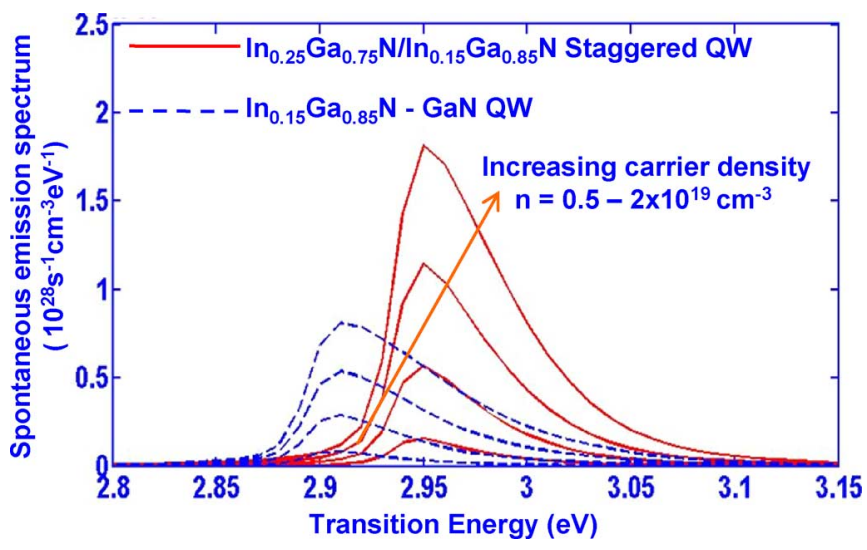

Fig. 3. Spontaneous recombination rate spectra of $25-\AA$ conventional $\mathrm{In}_{0.15} \mathrm{Ga}_{0.85} \mathrm{~N}$ QW and $15-\AA$ staggered $\mathrm{In}_{025} \mathrm{Ga}_{0.75} \mathrm{~N}-\mathrm{In}_{0.15} \mathrm{Ga}_{0.85} \mathrm{~N}$ QW for $n=0.5,1,1.5$, and $2 \times 10^{19} \mathrm{~cm}^{-3}$.

that recombines radiatively in the $\mathrm{QW}$ active region. At $n \sim$ $2 \times 10^{19} \mathrm{~cm}^{-3}$, the peak spontaneous emission rate of the staggered InGaN QW structure is found to be approximately 2 times higher than that of the conventional one, corresponding to higher radiative recombination rate expected from the improvement of electron-hole wavefunction overlap. As carrier density is increased, blueshift of the peak emission wavelength also takes place. The wavelength blueshift in the staggered InGaN QW appears to be larger than that in the conventional structure, which can be attributed to thinner staggered InGaN QW leading to faster state-filling than in the conventional case.

The carrier density dependence of recombination rate per unit volume of conventional and staggered InGaN QW can be calculated as follows. First, the $r_{\mathrm{sp}}(\hbar \omega)$ is integrated over all frequencies to result in spontaneous recombination rate per unit volume $\left(L_{\mathrm{sp}}\right)$ at a particular carrier density $n_{o}$

$$
L_{\mathrm{sp}}\left(n_{o}\right)=\int r_{\mathrm{sp}}(\hbar \omega) d \omega \text { at } n=n_{o} .
$$

Note that the spontaneous emission rate per unit volume $\left(L_{\mathrm{sp}}\right)$ can be related to the carrier density in the QW $(n)$ with the following phenomenological approximation:

$$
L_{\mathrm{sp}}(n) \cong B \cdot n \cdot p=B \cdot n^{\beta}
$$

where $B$ is the bimolecular recombination constant for radiative transition. At high carrier injection level, electron density is assumed as equal to that of hole $(n \cong p)$. Fig. 4 illustrates integrated spontaneous recombination rate per unit volume $L_{\mathrm{sp}}$ of conventional and staggered InGaN QW plotted against carrier density, $n$. Equation indicating the dependence of $L_{\mathrm{sp}}$ on $n$ for each plot is also shown. From Fig. 4, we found that $\beta<2$ for both QWs up to $n=2 \times 10^{19} \mathrm{~cm}^{-3}$. For the case of $n=2 \times 10^{19} \mathrm{~cm}^{-3}$, the staggered InGaN QW structure exhibits close to two times higher integrated spontaneous emission rate of $L_{\mathrm{sp}}=1.36 \times 10^{27} \mathrm{~s}^{-1} \mathrm{~cm}^{-3}$ than that of the conventional one of $L_{\mathrm{sp}}=7.62 \times 10^{26} \mathrm{~s}^{-1} \mathrm{~cm}^{-3}$. The integrated spontaneous emission rate $\left(L_{\mathrm{sp}}\right)$ of the staggered InGaN QW also exhibits increase in the exponent term $\left(\beta_{\mathrm{st}}=1.923\right)$ dependent with carrier density compared to that of the conventional QW $\left(\beta_{\text {con }}=1.8867\right)$. The increase in $\beta$ for staggered InGaN 


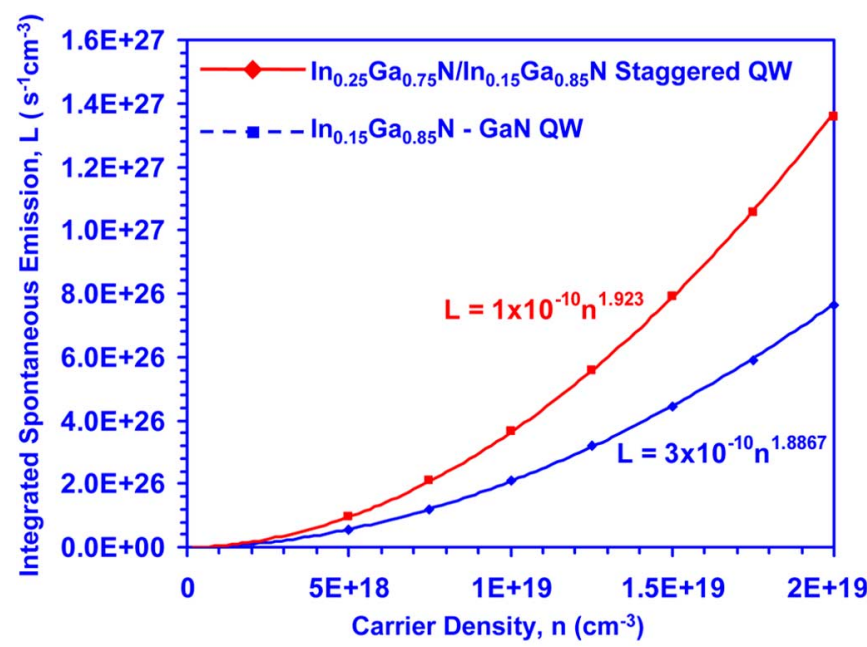

Fig. 4. Spontaneous recombination rate per unit volume, $L_{\mathrm{sp}}$ of $25 \AA$ conventional $\mathrm{In}_{0.15} \mathrm{Ga}_{0.85} \mathrm{~N}$ QW and $15 \AA$ staggered $\operatorname{In}_{025} \mathrm{Ga}_{0.75} \mathrm{~N}-\mathrm{In}_{0.15} \mathrm{Ga}_{0.85} \mathrm{~N}$ QW.

QW can be attributed to the higher transition matrix element, which leads to higher rate of increase for the integrated spontaneous emission rate with increasing carrier injection. However, for the high carrier density operation $\left(n>3 \times 10^{19} \mathrm{~cm}^{-3}\right)$, the enhancement ratio of the integrated spontaneous emission rate $\left(L_{\mathrm{sp}}\right)$ for staggered InGaN QW will be reduced due to carrier screening effect.

Experiments had been conducted to compare the optical properties of staggered and conventional InGaN QW, with emission wavelength at $420-430 \mathrm{~nm}$. Both the conventional and staggered InGaN QWs samples were prepared by VEECO P-75 vertical metalorganic chemical vapor deposition (MOCVD) reactor on 2.5- $\mu \mathrm{m}$-thick undoped $\mathrm{GaN}\left(\mathrm{T}_{g}=1080^{\circ} \mathrm{C}\right)$ grown on $c$-plane sapphire, employing a low temperature $30-\mathrm{nm}$ $\mathrm{GaN}$ buffer layer $\left(\mathrm{T}_{\mathrm{g}}=535^{\circ} \mathrm{C}\right)$. The conventional $\mathrm{QW}$ structure consists of four periods of $25-\AA \mathrm{In}_{0.15} \mathrm{Ga}_{0.85} \mathrm{~N} \mathrm{QW}$, while the staggered QW structure is formed by four periods of 7.5- $\AA \operatorname{In}_{0.25} \mathrm{Ga}_{0.75} \mathrm{~N} / 7.5-\AA \mathrm{In}_{0.15} \mathrm{Ga}_{0.85} \mathrm{~N}$ layers. We employed $12-\mathrm{nm} \mathrm{GaN}$ barriers in both QW structures. All the active and barrier regions in the structures studied were grown at a temperature of $720^{\circ} \mathrm{C}$. Trimethyl Gallium (TMGa) was used as the group III precursor for the high-temperature undoped GaN virtual substrate, while Trimethyl Indium (TMIn) and Triethyl Gallium (TEGa) were used for the QW structures. $\mathrm{NH}_{3}$ gas was used as the group $\mathrm{V}$ source. The composition and growth rate of the $\operatorname{In}_{x} \mathrm{Ga}_{1-x} \mathrm{~N}$ alloy were calibrated individually by high-resolution X-ray diffraction (XRD) measurements using 4-periods of $\operatorname{In}_{x} \mathrm{Ga}_{1-x} \mathrm{~N}-\mathrm{GaN}$ superlattices. The staggered InGaN QW is then realized using growth conditions (flow rates, V-III, growth rate, duration, temperature, and pressure) obtained from this calibration. The In-contents and thicknesses for QW structures are verified by comparing the peak photoluminescence wavelengths with the data from XRD calibration and numerical model.

Room temperature cathodoluminescence (RT-CL) measurements were performed utilizing $10 \mathrm{keV}$ electron beam with $1 \mu \mathrm{A}$ of current over a raster scan area size of $800 \times 600 \mu \mathrm{m}^{2}$, with integration time of $0.1 \mathrm{~s}$. The CL emission wavelengths

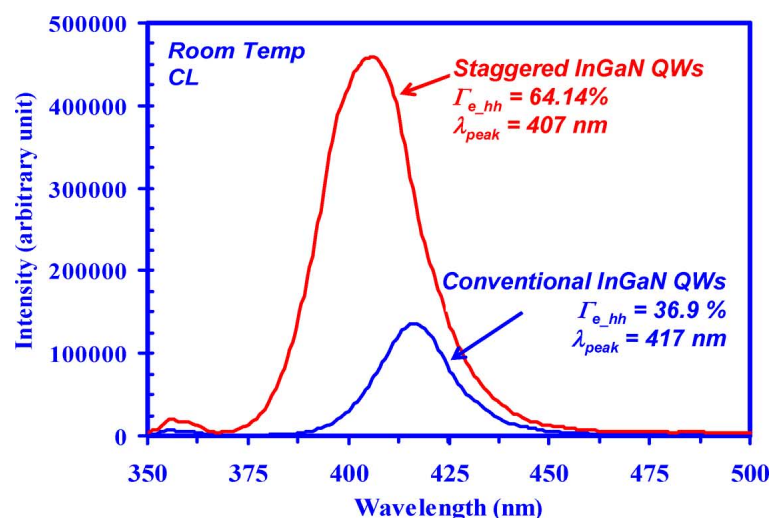

(a)

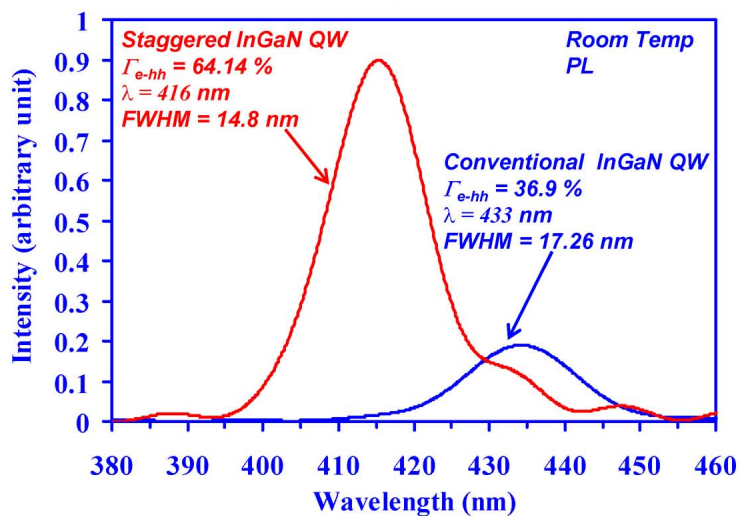

(b)

Fig. 5. Room-temperature (a) CL and (b) PL spectra of staggered 7.5- $\AA$ $\mathrm{In}_{0.25} \mathrm{Ga}_{0.75} \mathrm{~N}-7.5-\AA \mathrm{In}_{0.15} \mathrm{Ga}_{0.85} \mathrm{~N}$ QW and $25-\AA$ conventional type-I $\mathrm{In}_{0.25} \mathrm{Ga}_{0.75} \mathrm{~N}$ QW.

for staggered QW and conventional QW are measured as 407 and $417 \mathrm{~nm}$, respectively. The CL emission of both the staggered and conventional QWs were blue-shifted by $10-15 \mathrm{~nm}$ in comparison to those of the photoluminescent (PL) wavelengths, presumably due to larger carrier screening effect in CL measurements. As shown in Fig. 5(a), the staggered QW structure exhibited an increase of CL peak intensity and integrated luminescence intensity by a factor of 3.37 and 4.15 times, respectively, in comparison to those of the conventional QW.

Room-temperature PL (RT-PL) measurements were also performed on both samples using $325-\mathrm{nm} \mathrm{He}-\mathrm{Cd}$ lasers. As shown in Fig. 5(b), the PL peak emission wavelengths $\left(\lambda_{\text {peak }}\right)$ of the staggered $\mathrm{QW}$ and conventional $\mathrm{QW}$ were measured as 420-nm and 430-nm, respectively. The peak PL and integrated PL luminescence intensities for the staggered $\mathrm{In}_{0.25} \mathrm{Ga}_{0.75} \mathrm{~N}-\mathrm{In}_{0.15} \mathrm{Ga}_{0.85} \mathrm{~N}$ QWs exhibited improvement by a factor of 4.74 and 4.39 times, respectively, in comparison to those of the conventional $\mathrm{In}_{0.25} \mathrm{Ga}_{0.75} \mathrm{~N}$ QW. It is also important to point out that integrated luminescence improvement in the staggered InGaN QW is not accompanied by increased linewidth, rather it is attributed to higher peak intensity at the same excitation laser power. This is consistent with the fact that the measurements were conducted at the same temperature employing identical optical excitation power to facilitate direct spectrum comparison. The PL full-width at half-maximum (FWHM) for staggered InGaN QWs is measured as $14.8 \mathrm{~nm}$ 


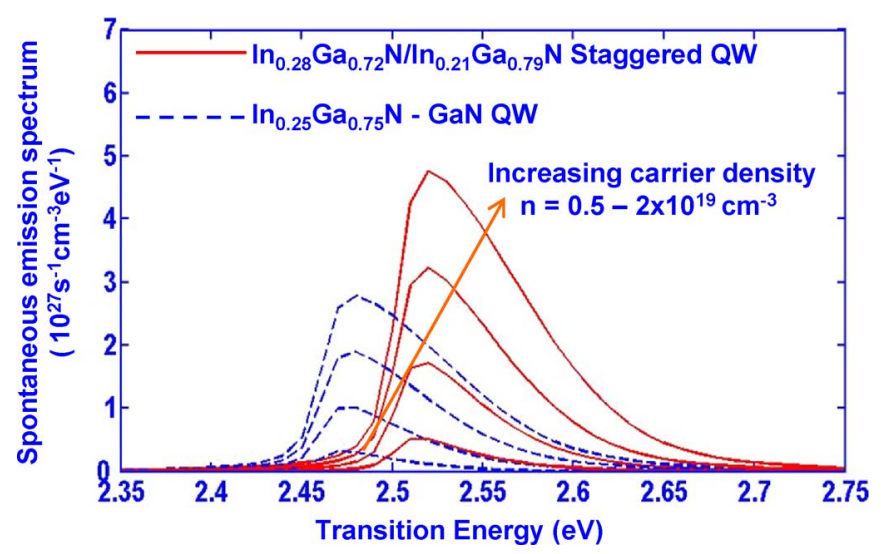

Fig. 6. Spontaneous recombination rate spectra of $27-\AA$ conventional $\mathrm{In}_{0.25} \mathrm{Ga}_{0.75} \mathrm{~N} \mathrm{QW}$ and $26-\AA$ staggered $\mathrm{In}_{028} \mathrm{Ga}_{0.72} \mathrm{~N}-\mathrm{In}_{0.21} \mathrm{Ga}_{0.79} \mathrm{~N} \mathrm{QW}$ for $n=0.5,1,1.5$, and $2 \times 10^{19} \mathrm{~cm}^{-3}$.

(106.2 meV), which is comparable to that of the conventional InGaN QW (FWHM $=17.3 \mathrm{~nm}$ or $113.8 \mathrm{meV}$ ). The comparable PL FWHMs indicate that the material qualities of both the staggered and conventional InGaN QWs are similar.

\section{B. Comparison in the Green Regime $\left(\lambda_{\text {peak }}=500-510 \mathrm{~nm}\right)$}

To further extend the concept of radiative recombination enhancement, we investigated conventional and staggered InGaN QW structures emitting at $\lambda_{\text {peak }}=500-510 \mathrm{~nm}$. The calculated spontaneous emission rate spectrum for this wavelength regime is shown in Fig. 6 for carrier density $n$ from $5 \times 10^{18} \mathrm{~cm}^{-3}$ up to $2 \times 10^{19} \mathrm{~cm}^{-3}$. The structures used in this study are $27-\AA$ conventional $\mathrm{In}_{0.25} \mathrm{Ga}_{0.75} \mathrm{~N}$ QW and staggered 13- $\AA \mathrm{In}_{0.28} \mathrm{Ga}_{0.72} \mathrm{~N}-13-\AA \mathrm{In}_{0.21} \mathrm{Ga}_{0.79} \mathrm{~N}$ QW. Similar to the 420-nm regime case (Fig. 3), wavelength blueshift is observed with increasing carrier density due to state-filling. Similar to the 420-nm regime case (Fig. 3), wavelength blueshift is observed in Fig. 6 with increasing carrier density due to state-filling for low carrier density from $n=5 \times 10^{18} \mathrm{~cm}^{-3}$ up to $n=2 \times 10^{19} \mathrm{~cm}^{-3}$. Note that for high carrier density operation $\left(n>3 \times 10^{19} \mathrm{~cm}^{-3}\right)$ in laser devices, wavelength blueshift from carrier screening will be significant. At $\boldsymbol{k}=0$, wavefunction overlap for $E_{C 1}-E_{\mathrm{HH} 1}$ transition $\left(\Gamma_{\mathrm{e}-\mathrm{hh}}\right)$ for the conventional and staggered InGaN QW case is $24.4 \%$ and $32.2 \%$, respectively. The improvement in the peak spontaneous emission spectrum of the staggered InGaN QW was found to be approximately 1.7 times higher than that of the conventional one for carrier density $n=2 \times 10^{19} \mathrm{~cm}^{-3}$. One discerning feature is that the nominal calculated values of the spontaneous emission spectrum at this $\lambda_{\text {peak }}=500-515 \mathrm{~nm}$ regime is approximately four times lower than that of the 420-nm regime. This is because, to achieve long emission wavelength, one needs to utilize high In-content leading to high polarization field and much lower resulting wavefunction overlap $\Gamma_{\mathrm{e} \_h h}$. Spontaneous recombination rate is thus inevitably reduced significantly. A thick QW to minimize quantum confinement further exacerbates this low recombination rate at the long wavelength regime of green and beyond.

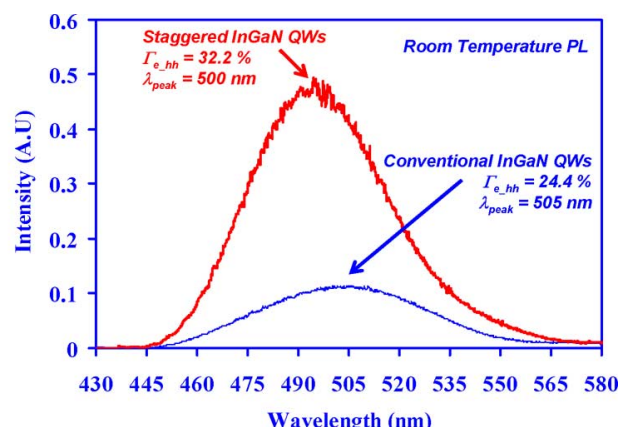

(a)

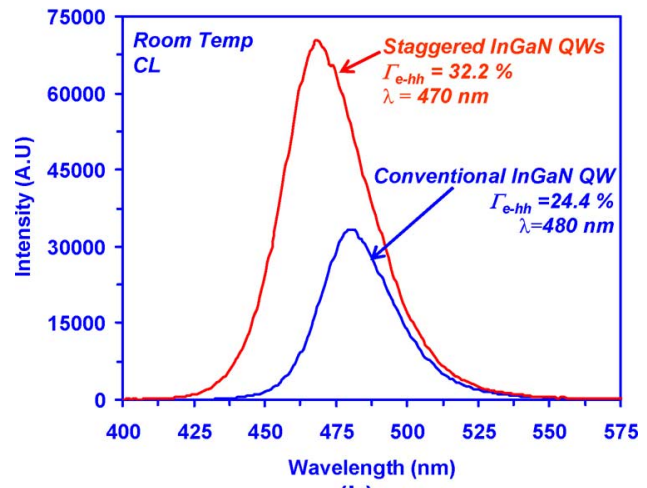

(b)

Fig. 7. Room-temperature (a) PL spectra and (b) CL spectra of of staggered $13-\AA \mathrm{In}_{0.28} \mathrm{Ga}_{0.72} \mathrm{~N}-13 \AA \mathrm{In}_{0.21} \mathrm{Ga}_{0.79} \mathrm{~N} \mathrm{QW}$ and $27-\AA$ conventional $\mathrm{In}_{0.26} \mathrm{Ga}_{0.74} \mathrm{~N} \mathrm{QW}$.

As shown in Fig. 7(a), the staggered $\mathrm{In}_{0.28} \mathrm{Ga}_{0.72} \mathrm{~N}-\mathrm{In}_{0.21} \mathrm{Ga}_{0.79} \mathrm{~N} \quad \mathrm{QW}$ exhibited improvements in the peak PL and the total integrated PL luminescence intensity by 4.48 and 3.54 times, respectively. In Fig. 7(b), the staggered QW structure exhibited an increase in CL peak intensity and integrated luminescence intensity by a factor of 2.1 and 2.43 times, respectively, in comparison to those of the conventional QW. The PL and CL improvements were higher than what were predicted from the increase in overlap $\Gamma_{\text {e_hh }}$ at $\boldsymbol{k}=0$ alone and from our spontaneous recombination rate spectrum calculation. The improvements could thus be presumably due to: 1) better materials quality in staggered InGaN QW and 2) lower "effective" QW thickness in the staggered QW structure, as compared to that of the conventional InGaN QW, leading to higher carrier concentration in the QW and higher radiative efficiency, hence further enhancing the intensity improvement.

\section{COMPARISON OF CONVENTIONAL AND STAGGERED INGAN QW LEDS $\left(\lambda_{\text {peak }} \sim 460 \mathrm{~nm}\right)$}

To assess the staggered QWs for device applications, we realized two LED structures $\left(\lambda_{\text {peak }}=455-465 \mathrm{~nm}\right.$ ) utilizing 1) four periods of staggered QWs of $12-\AA \operatorname{In}_{0.25} \mathrm{Ga}_{0.75} \mathrm{~N}-12-\AA$ $\operatorname{In}_{0.15} \mathrm{Ga}_{0.85} \mathrm{~N}$ layers $\left(\Gamma_{\mathrm{e}-\mathrm{hh}}=43.1 \%\right)$, and 2 ) four periods of $27-\AA$ conventional $\operatorname{In}_{0.21} \mathrm{Ga}_{0.79} \mathrm{~N} \mathrm{QW}\left(\Gamma_{\mathrm{e}-\mathrm{hh}}=27.4 \%\right)$ as the active regions of each LED. Both structures were grown on $2.5-\mu \mathrm{m} \mathrm{n}$-GaN template $\left(n=3 \times 10^{18} \mathrm{~cm}^{-3}\right)$ on $c$-plane sapphire substrates. The growth procedure of the staggered InGaN QW LED structure is described in Fig. 8. We start with hightemperature growth of $\mathrm{n}-\mathrm{GaN}$ layer at around $1090{ }^{\circ} \mathrm{C}$ using 


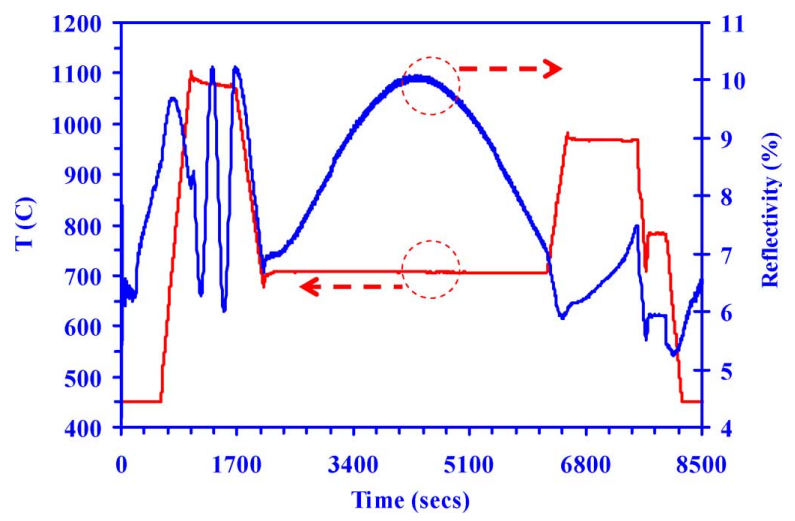

Fig. 8. Temperature and reflectivity profile taken in situ during the growth of staggered 12- $\AA \mathrm{In}_{0.25} \mathrm{Ga}_{0.75} \mathrm{~N}-12-\AA \mathrm{In}_{0.15} \mathrm{Ga}_{0.85} \mathrm{~N}$ QWs LED.

TMGa and $\mathrm{NH}_{3}$ gas as the group III and group $\mathrm{V}$ precursor, respectively, and $\mathrm{H}_{2}$ as the carrier gas. Next, the temperature is ramped down for the low-temperature growth of four periods of staggered InGaN-GaN QW at $705^{\circ} \mathrm{C}$ utilizing TEGa and TMIn as the group-III precursor and $\mathrm{N}_{2}$ carrier gas. Following that, the growth of the upper p-doped $\mathrm{GaN}$ layer is performed at $970{ }^{\circ} \mathrm{C}$. The thickness of this upper p-type GaN layer is approximately $0.2 \mu \mathrm{m}$. Subsequently, p-dopant activation anneal is performed at $780{ }^{\circ} \mathrm{C}$ for $5 \mathrm{~min}$ in $\mathrm{N}_{2}$ ambient. The acceptor level for the $\mathrm{p}-\mathrm{GaN}$ layer is measured as $3 \times 10^{17} \mathrm{~cm}^{-3}$.

Continuous-wave $(\mathrm{CW})$ power measurements were performed at room temperature. Fig. 9 shows that the output power is linear as function of driving current up to $100 \mathrm{~mA}$ for both LEDs with injection area of $1 \mathrm{~mm}^{2}$. Staggered QW LEDs exhibited an improvement in output power by 11.2 times at current level of $100 \mathrm{~mA}$. The measured significant enhancement is larger than that predicted theoretically, and several possible factors may contribute to this improvement (in addition to the effect from improved overlap $\Gamma_{\text {e_hh }}$ ), such as: 1) improvement in the material quality of the staggered $\mathrm{InGaN}$ QWs leading to reduction in monomolecular recombination rate and/or 2) improvement in the carrier confinement in the staggered InGaN QWs. Further studies and optimizations are still required to elucidate more insights into the physics of polarization engineering of nitride-based active regions, in particular recombination analysis (i.e., power dependent cathodoluminescence and scanning near-field optical microscopy PL) and structural analysis (ie. transmission electron microscopy) of the staggered InGaN QW are needed to clarify the factors leading to this improvement.

\section{SUMMARY}

In summary, polarization band engineering using staggered InGaN QWs layers for improved wavefunction overlap $\left(\Gamma_{\mathrm{e}_{-} \mathrm{hh}}\right)$ leads to significant enhancement of radiative recombination rate. The calculated spontaneous emission spectra and radiative recombination rate using 6-band $\boldsymbol{k} . \boldsymbol{p}$ band structure formalism show staggered InGaN QW lead to significantly improved active region, which is applicable for achieving high radiative efficiency III-nitride LEDs. Staggered InGaN QWs LEDs were

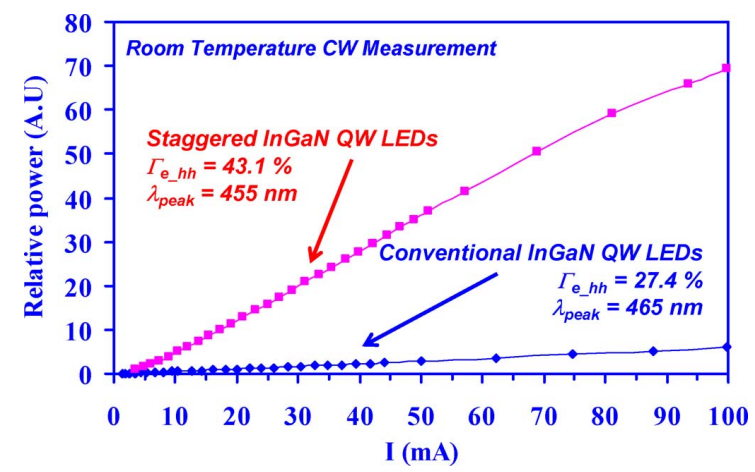

Fig. 9. Relative light output power versus injected current for staggered 12- $\AA \mathrm{In}_{0.25} \mathrm{Ga}_{0.75} \mathrm{~N}-12-\AA \mathrm{In}_{0.15} \mathrm{Ga}_{0.85} \mathrm{~N}$ QW LED and $27-\AA$ conventional $\mathrm{In}_{0.21} \mathrm{Ga}_{0.79} \mathrm{~N} \mathrm{QW}$ LED.

grown by MOCVD, and the optical properties and device properties were compared with those of conventional InGaN QW LEDs. Improvements in PL peak luminescence intensity and integrated luminescence by a factor of 4.74 and 4.39 times (and 4.48 and 3.54 times), respectively, had been experimentally demonstrated for staggered InGaN QWs active regions emitting at $\lambda_{\text {peak }}=420-430 \mathrm{~nm}$ (and $\lambda_{\text {peak }}=500-505 \mathrm{~nm}$ ), which are in good agreement with the predicted theory. Preliminary LEDs device had also been fabricated utilizing staggered InGaN QW emitting in $\lambda_{\text {peak }}=455-465 \mathrm{~nm}$, resulting in almost an order of magnitude improvement in the output power of the devices. The use of polarization control for enhancing the $\Gamma_{\text {e_hh }}$ of the InGaN-based active regions can hence be potentially applicable for high-efficiency LEDs and low-threshold nitride-based lasers.

\section{REFERENCES}

[1] S. Nakamura, M. Senoh, N. Iwasa, S. Nagahama, T. Yamada, and T. Mukai, "Superbright green InGaN single-quantum-well-structure light-emitting diode,” Jpn. J. Appl. Phys., vol. 34, pp. L1332-L1335, Oct. 1995.

[2] S. Nakamura, M. Senoh, S. Nagahama, N. Iwasa, T. Yamada, T. Matshushita, H. Kiyoku, and Y. Sugimoto, "InGaN-based multi-quantumwell-structure laser diodes," Jpn. J. Appl. Phys., vol. 35, pp. L74-L76, Jan. 1996.

[3] S. Chhajed, Y. Xi, Y.-L. Li, T. Gessmann, and E. F. Schubert, "Influence of junction temperature on chromaticity and color-rendering properties of trichromatic white-light sources based on light-emitting diodes," J. Appl. Phys., vol. 97, pp. 054506-1-054506-8, Feb. 2005.

[4] W. Zhao, Y. Li, T. Detchprohm, and C. Wetzel, "The quantum efficiency of green GaInN/GaN light emitting diodes," Phys. Stat. Sol. (c), vol. 4, pp. 9-12, Jan. 2007

[5] R. H. Horng, C. C. Yang, J. Y. Wu, S. H. Huang, C. E. Lee, and D S. Wuu, "GaN-based light-emitting diodes with indium tin oxide texturing window layers using natural lithography," Appl. Phys. Lett., vol. 86, pp. 221101-1-221101-3, May 2005.

[6] J. S. Cabalu et al., "Visible light-emitting diodes grown by plasma assisted molecular beam epitaxy on hydride vapor-phase epitaxy GaN templates and the development of dichromatic (Phosphorless) white LEDs," in Proc. Mater. Res. Soc. Symp., 2006, vol. 892, pp. 0892FF12-04.1-0892-FF12-04.6.

[7] J. Zhang, J. Yang, G. Simin, M. Shatalov, M. A. Khan, M. S. Shur, and R. Gaska, "Enhanced luminescence in InGaN multiple quantum wells with quaternary AlInGaN barriers," Appl. Phys. Lett., vol. 77, pp. 2668-2670, Oct. 2000.

[8] T. Deguchi, K. Sekiguchi, A. Nakamura, T. Sota, R. Matsuo, S. Chichibu, and S. Nakamura, "Quantum-confined stark effect in an AlGaN/GaN/AlGaN single quantum well structure," Jpn. J. Appl. Phys., vol. 38, pp. L914-L916, Aug. 1999. 
[9] F. Bernardini, V. Fiorentini, and D. Vanderbilt, "Spontaneous polarization and piezoelectric constants of III-V nitrides," Phys. Rev. B, vol. 56, pp. R10024-R10027, Oct. 1997.

[10] F. Bernardini and V. Fiorentini, "Macroscopic polarization and band offsets at nitride heterojunctions," Phys. Rev. B, vol. 57, pp. R9427-R9430, Apr. 1998.

[11] S. Nagahama, Y. Sugimoto, T. Kozaki, and T. Mukai, "Recent progress of AlInGaN laser diodes," Proc. SPIE, vol. 5738, pp. 57-62, Apr. 2005.

[12] C. Wetzel, S. Kamiyama, H. Amano, and I. Akasaki, "Optical absorption in polarized $\mathrm{Ga}_{1-x} \mathrm{In}_{x} \mathrm{~N} / \mathrm{GaN}$ quantum wells," Jpn. J. Appl. Phys., vol. 41, pp. 11-14, Jan. 2002.

[13] R. A. Arif, Y.-K. Ee, and N. Tansu, "Polarization engineering via staggered InGaN quantum wells for radiative efficiency enhancement of light emitting diodes," Appl. Phys. Lett., vol. 91, pp. 091110-1-091110-3, Aug. 2007.

[14] L. A. Coldren and S. W. Corzine, Diode Lasers and Photonic Integrated Circuits. New York: Wiley, 1995.

[15] J. Singh, Electronic and Optoelectronic Properties of Semiconductor Structures. Cambridge, U.K.: Cambridge Univ. Press, 2003.

[16] S. L. Chuang and C. S. Chang, "A band-structure model of strained quantum-well wurtzite semiconductors," Semicond. Sci. Technol., vol. 12, pp. 252-263, Mar. 1997.

[17] S. Chuang, "Optical gain of strained wurtzite GaN quantum-well lasers," IEEE J. Quantum Electron., vol. 32, no. 10, pp. 1791-1800, Oct. 1996.

[18] I. Vurgaftman and J. R. Meyer, "Band parameters for nitrogen-containing semiconductors," J. Appl. Phys., vol. 94, pp. 3675-3696, Sep. 2003.

[19] J. Piprek, Semiconductor Optoelectronic Devices: Introduction to Physics and Simulation. London, U.K.: Academic Press, 2003.

[20] Y. C. Yeo, T. C. Chong, M. F. Li, and W. J. Fan, "Analysis of optical gain and threshold current density of wurtzite InGaN/GaN/AlGaN quantum well lasers," J. Appl. Phys., vol. 84, pp. 1813-1819, Aug. 1998.

[21] J. Wu, W. Walukiewicz, W. Shan, K. M. Yu, J. W. Ager, III, S. X. Li, E. E. Haller, H. Lu, and W. J. Schaff, "Temperature dependence of the fundamental bandgap of InN," J. Appl. Phys., vol. 94, pp. 4457-4460, Oct. 2003.

[22] O. Ambacher, J. Majewski, C. Miskys, A. Link, M. Hermann, M. Eickhoff, M. Stutzmann, F. Bernardini, V. Fiorentini, V. Tilak, W. J. Schaff, and L. F. Eastman, "Pyroelectric properties of $\mathrm{Al}(\mathrm{In}) \mathrm{GaN} / \mathrm{GaN}$ heteroand quantum well structures," J. Phys.: Condens. Matter, vol. 14, pp. 3399-3434, Mar. 2002.

[23] A. Chakraborty, B. A. Haskell, S. Keller, J. S. Speck, S. P. Denbaars, S. Nakamura, and U. K. Mishra, "Demonstration of nonpolar $m$-plane InGaN/GaN light-emitting diodes on free-standing $m$-plane GaN substrates," Jpn. J. Appl. Phys., vol. 44, pp. L173-L175, Jan. 2005.

[24] Y.-L. Li, T. Gessmann, E. F. Schubert, and J. K. Sheu, "Carrier dynamics in nitride-based light-emitting p-n junction diodes with two active regions emitting at different wavelengths," J. Appl. Phys., vol. 94, pp. 2167-2172, Aug. 2003.

[25] N. F. Gardner, G. O. Muller, Y. C. Shen, G. Chen, S. Watanabe, W. Gotz, and M. R. Krames, "Blue-emitting InGaN-GaN double-heterostructure light emitting diodes reaching maximum quantum efficiency above $200 \mathrm{~A} / \mathrm{cm}^{2}$," Appl. Phys. Lett., vol. 91, Dec. 2007.

[26] Y. C. Shen, G. O. Mueller, S. Watanabe, N. F. Gardner, A. Munkholm, and M. R. Krames, "Auger recombination in InGaN measured by photoluminescence," Appl. Phys. Lett., vol. 91, p. 141101, Oct. 2007.

[27] P. G. Eliseev, M. Osinski, Li Hua, and I. V. Akimova, "Recombination balance in green-light-emitting GaN/InGaN/AlGaN quantum wells," Appl. Phys. Lett., vol. 75, no. 24, pp. 3838-3840, Dec. 1999.

[28] S. Nagahama, Y. Sugimoto, T. Kozaki, and T. Mukai, "Recent progress of AlInGaN laser diodes," Proc. SPIE, vol. 5738, pp. 57-62, Apr. 2005.

[29] H. Y. Ryu, K. H. Ha, S. N. Lee, T. Jang, J. K. Son, H. S. Paek, Y. J. Sung, H. K. Kim, K. S. Kim, O. H. Nam, Y. J. Park, and J. I. Shim, "High-performance blue InGaN laser diodes with single-quantum-well active layers," IEEE Photon. Technol. Lett., vol. 19, pp. 1717-1719, 2007.

[30] M. Röwe, P. Michler, J. Gutowski, V. Kümmler, A. Lell, and V. Härle, "Influence of the carrier density on the optical gain and refractive index change in InGaN laser structures," Phys. Stat. Solidi (a), vol. 200, pp. 135-138, Sep. 2003.
Ronald A. Arif received the B.S. degree (first-class hons) in materials engineering from Nanyang Technological University, Singapore, in 2002, the M.S. degree in electrical engineeringfrom Lehigh University, Bethlehem, PA, in 2005, where he is currently pursuing the Ph.D. degree in electrical engineering in the Department of Electrical and Computer Engineering.

He was a Process Engineer with Agilent Technologies, Singapore from 2002 to 2003, where his main responsibilities included growth of III-V semiconductor materials for optoelectronics devices. His research areas cover the fundamental device physics, technology, epitaxy (MOCVD), and fabrication of semiconductor optoelectronics devices. His research works include fundamental studies and novel approaches to improve radiative efficiency of visible gain media based on III-nitride semiconductor nanostructures for high-efficiency LEDs and lasers, in particular for solid state lighting. He has published in numerous refereed journal and conference publications (more than 40). He previously worked on novel $\mathrm{SbN}$-based (i.e., InGaAsSbN) interdiffused quantum-well gain media for 1300-1550-nm lasers and interdiffused InGaAsP quantum dots for 980-nm EDFA pump lasers.

Hongping Zhao received the B.S. degree in physics from Nanjing Normal University, Nanjing, China, in 2003 and the M.S. degree in electrical engineering from Southeast University, China, in 2005, respectively. She is currently pursuing the Ph.D. degree candidate in the Electrical and Computer Engineering Department at Lehigh University, Bethlehem, PA.

Her research areas cover device physics, epitaxial growth, and fabrication of semiconductor optoelectronics devices based on semiconductor nanostructures. Current works include fundamental studies and approaches to improve gain and spontaneous emission of visible gain media based on III-nitride semiconductors for high performance lasers.

Yik-Khoon Ee received the B.S. degree (first-class hons) in electrical and electronic engineering from Nanyang Technological University, Singapore, in 2003, and the M.S. degree from Lehigh University, Bethlehem, PA, in 2007, where he is currently pursuing the Ph.D. degree in the Department of Electrical and Computer Engineering .

He was with Denselight Semiconductors and Agilent Technologies. His research works are in the field of semiconductor optoelectronics, MOCVD epitaxy, device fabrication of III-nitride LEDs. His research focus also includes the growth of InGaN quantum dots, nanoheteroepitaxy, and optimization of light extraction efficiency in III-Nitride LEDs.

Mr. Ee was a recipient of the Singapore Economic Development Board Photonics Scholarship and the Sherman Fairchild Fellowship.

Nelson Tansu was born in October 1977. He received the B.S. degrees (with highest distinction) in applied mathematics, electrical engineering, and physics in 1998 and the Ph.D. degree in electrical engineering from the University of Wisconsin-Madison in 2003.

Since July 2003, he has been an Assistant Professor in the Department of Electrical and Computer Engineering (ECE) and the Center for Optical Technologies (COT) at Lehigh University, Bethlehem, PA. Since April 2007, he has been appointed as the Peter C. Rossin Assistant Professor of Electrical and Computer Engineering at Lehigh University. His research works cover the theoretical and experimental aspects of the physics of semiconductor optoelectronics materials and devices, the physics of low-dimensional semiconductor (nanostructure), and MOCVD epitaxy and device fabrications of III-V-nitride and III-nitride semiconductor optoelectronics devices on GaAs, InP, and GaN substrates. His teaching interests are in the areas of optoelectronics and photonics, semiconductor physics, applied quantum mechanics, and engineering electromagnetism. He has published widely in numerous refereed international journal and conference publications (more than 129), and he also currently holds several U.S. patents. He had served several times as a panel member for U.S. National Science Foundation, U.S. Department of Defense, and other agencies in U.S. and abroad. He has also given numerous lectures, seminars, and invited talks (more than 30) in universities, research institutions, and conferences in the U.S., Canada, Europe, and Asia.

Dr. Tansu was a recipient of the Bohn Scholarship, the WARF Graduate University Fellowship, the Vilas Graduate University Fellowship, and the Graduate Dissertator Travel Funding Award, the 2003 Harold A. Peterson ECE Best Research Award (First Prize) at the University of Wisconsin-Madison. 